\title{
INFORME ${ }^{1}$
}

\section{Avances en simplificación administrativa y administración electrónica}

\section{INTRODUGGIÓN Y GUESTIONES GENERALES}

A punto de finalizar 2019, el Consejo de Gobierno de la Junta de Andalucía ha aprobado el Decreto 622/2019, de 27 de diciembre, de administración electrónica, simplificación de procedimientos y racionalización organizativa de la Junta de Andalucía (BOJA 250, de 31 de diciembre), que está llamado a ser una pieza importante del Derecho Administrativo propio de la Comunidad Autónoma de Andalucía².

1 Esta sección ha sido elaborada por MARÍA DEL CARMEN NÚÑEZ LOZANO, Catedrática de Derecho Administrativo, Centro de Investigación en Patrimonio Histórico, Cultural y Natural, Universidad de Huelva.

2 Basta con apreciar el catálogo de preceptos y disposiciones que deroga expresamente: a) El Decreto 260/1988, de 2 de agosto, por el que se desarrollan atribuciones para la racionalización administrativa de la Junta de Andalucía. b) El artículo 4.b) del Decreto 90/1993, de 13 de julio, por el que se asignan diversas funciones a determinados órganos administrativos de la Junta de Andalucía. c) El capítulo III y los artículos 22, 23 y 25 del Decreto 204/1995, de 29 de agosto, por el que se establecen medidas organizativas para los servicios administrativos de atención directa a los ciudadanos. d) El artículo 2.2 del Decreto 48/2002, de 12 de febrero, por el que se establece el Registro Territorial de la Propiedad Intelectual de la Comunidad Autónoma de Andalucía. e) El artículo 3 del Decreto 72/2003, de 18 de marzo, de medidas de Impulso de la Sociedad del Conocimiento en Andalucía. f) El Decreto 183/2003, de 24 de junio, por el que se regula la información y atención al ciudadano y la tramitación de procedimientos administrativos por medios electrónicos (Internet). g) El artículo 3.4 del Decreto 86/2004, de 2 de marzo, sobre Cofradías de Pescadores y sus Federaciones. h) El Decreto 68/2008, de 26 de febrero, por el que se suprime la aportación de la fotocopia de los documentos identificativos oficiales y del certificado de empadronamiento en los procedimientos administrativos de la Administración de la Junta de Andalucía y se establece la sede electrónica para la práctica de la notificación electrónica, a excepción de su artículo 4.1 en lo relativo a la creación de la sede electrónica. i) La Orden de la Consejería de Gobernación de 28 de julio de 1989, por la que se establecen los criterios de normalización de formularios y papel impreso y se crea el registro de formularios de la Junta de Andalucía. j) Los artículos 5 y 6 de la Orden de la Consejería de Gobernación, de 1 de diciembre de 1995, por la que se normalizan las características que han de reunir los sellos oficiales empleados en la actuación administrativa de la Junta de Andalucía. k) El capítulo II de la Orden de 10 de junio de 2005, por la que se regula el Registro Telemático Tributario dependiente de la Consejería de Economía y Hacienda y las notificaciones, certificados y transmisiones telemáticos en el ámbito de la Administración Tributaria de la Junta de Andalucía, referido al Registro Telemático Tributario. 1) La Orden de 24 de noviembre de 2005, por la que se establecen determinados procedimientos administrativos de la Consejería de Justicia y Administración Pública que podrán iniciarse por medios telemáticos. m) La Orden de la Consejería de Justicia y Administración Pública de 11 de octubre de 2006, por la que se establece la utilización de medios electrónicos para la expedición de copias autenticadas. n) La Orden de 27 de diciembre de 2006, por la que se regula la utilización de medios electrónicos a aplicativos de gestión de la Consejería de Justicia y Administración Pública. Téngase en cuenta que el capítulo II de la Orden de 10 de junio de 2005, por la 
Consta de 48 artículos, agrupados en ocho capítulos, ocho disposiciones adicionales, cuatro disposiciones transitorias, la disposición derogatoria, cuatro disposiciones finales ${ }^{3}$ y cuatro anexos ${ }^{4}$.

El objeto de la nueva norma es "establecer los principios generales y adoptar medidas de simplificación administrativa y racionalización organizativa, así como el uso de los medios electrónicos" por las entidades a las que es de aplicación (artículo 1.1), todo ello con las finalidades de mejorar la eficacia y la eficiencia en la actuación administrativa, garantizar los derechos de la ciudadanía en sus relaciones con las entidades a las que se aplica y preservar la seguridad jurídica (artículo 1.2).

El Decreto utiliza la palabra "ciudadanía" en sentido amplio, pues aclara el artículo 1.3 que "[a] los efectos de este decreto se entiende por ciudadanía todos aquellos a quienes se reconozca capacidad de obrar conforme al artículo 3 de la Ley 39/2015, de 1 de octubre, del Procedimiento Administrativo Común de las Administraciones Públicas" ${ }^{5}$. Aun siendo conscientes de la dificultad que presenta la selección

que se regula el Registro Telemático Tributario dependiente de la Consejería de Economía y Hacienda y las notificaciones, certificados y transmisiones telemáticos en el ámbito de la Administración Tributaria de la Junta de Andalucía, mantiene su vigencia hasta que se produzca la integración del Registro Telemático Tributario en el Registro Electrónico Único a que se refiere la disposición transitoria quinta.

3 En particular, la disposición final cuarta establece la entrada en vigor "en el plazo de tres meses a contar desde el día siguiente al de su publicación en el Boletín Oficial de la Junta de Andalucía", salvo "lo establecido en el artículo 45 en relación al Registro electrónico de apoderamientos, y lo dispuesto en el artículo 46.1 a) respecto de la identificación y firma electrónica por funcionario público habilitado", que producirá efectos el 2 de octubre de 2020 (si no se vuelve a modificar la disposición final séptima de la Ley 39/2015). Nótese que el Decreto no se sirve del margen proporcionado por la referida disposición final séptima de la Ley 39/2015 de manera que, respecto de las materias que regula, sólo aplaza la entrada en vigor de los aspectos indicados.

4 Sobre política de firma, sistemas de identificación admitidos no basados en certificados electrónicos, sistemas de firma admitidos no basados en certificados electrónicos y sistema de notificaciones electrónicas de la Administración de la Junta de Andalucía. Téngase en cuenta que los anexos pueden modificarse por orden de "la persona titular de la Consejería competente en materia de transformación digital" (disposición final segunda).

5 Durante la tramitación del Decreto, la Intervención General (informe de 30 de marzo de 2018) llamó la atención acerca del significado del vocablo "ciudadanía" que, sin mayores precisiones, se refiere a las personas físicas. Por esta razón, propuso que se incorporara al texto de la norma una definición más amplia del término; que se sustituyera por interesado o interesada; o que se efectuara una remisión al artículo 2.2 del Proyecto de Decreto por el que se aprueba el modelo de relación con la ciudadanía de la Administración de la Junta de Andalucía, entonces en tramitación ("personas físicas, personas jurídicas y entidades sin personalidad jurídica, que se relacionen o sean susceptibles de relacionarse con la Administración de la Junta de Andalucía, con los límites establecidos en el artículo 9"). También en el informe de la Secretaría General Técnica de la entonces Consejería de Economía, Hacienda y Administración Pública, de 6 de noviembre de 2018, se propuso que se explicitara que, por "ciudadanía", se entendían las personas físicas, personas jurídicas y entidades sin personalidad jurídica, que se relacionen o sean susceptibles de relacionarse con la Administración de la Junta de Andalucía y sus entidades instrumentales". 
de la palabra adecuada ${ }^{6}$, no cabe desconocer que parece que es el propósito de utilizar lo que se viene denominando un "lenguaje inclusivo" lo que motiva el empleo del término "ciudadanía", cuestionado también por el Consejo Consultivo en su informe de 19 de diciembre de 20197. En este sentido, causa sorpresa que en la definición de ciudadanía que se contiene en el artículo 1.3, que acabamos de reproducir, se haya incluido a "todos aquellos" y se haya olvidado a "todas aquellas".

Se aplica, en las relaciones que no son de derecho privado ${ }^{8}$, a: 1) la Administración de la Junta de Andalucía; 2) sus agencias administrativas; 3) las agencias públicas empresariales y las de régimen especial cuando, en ambos casos, ejercen potestades administrativas o se hace referencia específica a ellas en el Decreto; 4) a los consorcios que resulten adscritos a la Administración de la Junta de Andalucía de acuerdo con los criterios de prioridad establecidos en la Ley 40/2015, de 1 de octubre, de Régimen Jurídico del Sector Público, igualmente cuando ejercen potestades administrativas o se hace referencia específica a ellos en el Decreto (artículo 2.1). Se aplica también a las entidades instrumentales privadas de la Administración de la Junta de Andalucía, pero sólo en lo que se refiere a los portales de Internet que regulan los artículos 15 y 16 y a los registros electrónicos previstos en los artículos 47 y 48 de la disposición (artículo 2.2) ${ }^{9}$. Por lo que respecta a las entidades con régimen de independencia funcional o de especial autonomía (disposición adicional segunda

6 Pues el término "interesado" puede resultar insuficiente por su identificación con la titularidad de derechos e intereses legítimos. Y la palabra "persona" no incluye, precisamente, a las entidades sin personalidad jurídica.

7 En su dictamen de 19 de diciembre de 2019 recordó que "su significado preferente" se vincula a la nacionalidad, abogando por el empleo de la palabra "ciudadano".

8 Debe tenerse en cuenta lo dispuesto en la disposición adicional quinta, del siguiente tenor: "Las actuaciones y procedimientos de aplicación de los tributos, así como su revisión en vía administrativa se regirán por lo dispuesto en la normativa tributaria específica en los términos previstos en la disposición adicional primera de la Ley 39/2015, de 1 de octubre, del Procedimiento Administrativo Común de las Administraciones Públicas". Las singularidades tributarias explican que se habilite específicamente "a la persona titular de la Consejería competente en materia de hacienda para dictar cuantas disposiciones reglamentarias en materia tributaria sean precisas en desarrollo de este decreto, así como para coordinar y acordar las medidas necesarias para garantizar su efectiva ejecución e implantación" (apartado tercero de la disposición final segunda)

9 El informe del Gabinete Jurídico, de 19 de julio de 2019, apuntó la inclusión de las entidades instrumentales privadas cuando ejerzan potestades administrativas, dado que es el segundo criterio que la Ley 39/2015 utiliza para determinar la sujeción de esta clase de entidades a lo dispuesto en ella. Esta observación no fue tenida en cuenta porque la Ley 9/2007, de 22 de octubre, de la Administración de la Junta de Andalucía y la Ley 10/2005, de 31 de mayo, de Fundaciones de Andalucía, excluyen que las entidades instrumentales privadas puedan ejercer potestades administrativas (vid. la valoración del informe del Gabinete realizada por la Secretaría General para la Administración Pública, de 1 de octubre de 2019; antes, el informe de la Secretaría General Técnica de la entonces Consejería de la Presidencia, Administración Local y Memoria Democrática, de 17 de mayo de 2018, ya había llamado la atención acerca de estas circunstancias). 
de la Ley 9/2007, de 22 de octubre, de la Administración de la Junta de Andalucía), no se contempló inicialmente la aplicación del Decreto; tras la sugerencia formulada por el Consejo de Transparencia y Protección de Datos de Andalucía en el curso de la elaboración del Decreto, la disposición adicional tercera prevé finalmente que se rijan "por su normativa específica y supletoriamente por lo dispuesto en el presente decreto" $"$.

Aunque en el título del Decreto se incluya la expresión "racionalización organizativa" y su último capítulo se denomine "medidas organizativas", sustancialmente su contenido se reconduce a medidas de simplificación y de administración electrónica, estas últimas mucho más numerosas. En este sentido, resulta significativa la exposición de motivos del Decreto, que de forma repetida alude al par simplificación/ administración electrónica ${ }^{11}$. Claro está que, en la simplificación administrativa, entendida en sentido amplio, se puede entender incluida la racionalidad organizativa, como de hecho también se puede incluir en la racionalidad administrativa la simplificación administrativa y la administración electrónica ${ }^{12}$. La cuestión, en todo

10 En su informe de 30 de abril de 2018, el Consejo razonó que el desarrollo de la legislación básica de régimen jurídico de las administraciones públicas y del procedimiento administrativo común debía alcanzar también a la administración institucional, teniendo en cuenta que la autonomía orgánica y funcional no empecía a los objetivos de la futura norma. Al efecto, sugirió como solución técnica la ya adoptada en la disposición adicional segunda del Decreto 96/2017, de 27 de junio, por el que se regula la coordinación de la estrategia de imagen institucional de la Administración de la Junta de Andalucía, que es la que finalmente ha acogido el Decreto que nos ocupa.

11 De hecho, a pesar de que ya los primeros documentos del expediente de elaboración que se encuentran en el Portal de la Transparencia refieren como título de la futura norma el de "administración electrónica, simplificación de procedimientos y racionalización organizativa" (informe sobre la necesidad y oportunidad y memoria justificativa del proyecto de decreto y memoria económica, ambos de 20 de octubre de 2017), en el informe del Gabinete Jurídico se recoge como título del Proyecto de decreto el de "administración electrónica, simplificación de procedimientos y medios de racionalización administrativa”. Este lapsus que se advierte en el informe del Gabinete Jurídico podría revelar la conciencia de que, efectivamente, el contenido de la norma no se corresponde propiamente con la mención "racionalización organizativa" que luce en su título. En este sentido, también en el informe de evaluación del impacto de género, de 20 de octubre de 2017, se clasifica el contenido del Proyecto en "simplificación de procedimientos y racionalización administrativa" y "administración electrónica"; igualmente, el dictamen del Consejo Consultivo de Andalucía, de 19 de diciembre de 2019, reseña que “" t $]$ al y como se desprende de su título, el Proyecto de Decreto no se limita a regular la Administración Electrónica, sino que abarca diversas normas con finalidades de simplificación y racionalización administrativa y se extiende también a la regulación del cumplimiento de los principios de buena regulación en los procedimientos de elaboración de normas de la Junta de Andalucía”. Es posible, entonces, que con la inclusión de esa referencia simplemente se haya querido hacer muy visible el entronque del nuevo Decreto con el artículo 133 del Estatuto de Autonomía, que proclama los principios de "racionalidad organizativa" y de "simplificación de procedimientos".

12 Precisamente, la Comisión Interdepartamental de Coordinación y Racionalización Administrativa (artículo 5), que "tiene como finalidad el análisis de la situación, la planificación, coordinación y seguimiento de cuantas medidas se adopten para la racionalización y transformación continua de la Administración Pública", asume cometidos en ambas materias; es significativa también su composición. 
caso, no merece mayor detenimiento, aunque sí explica que en la exposición que realizamos a continuación se siga el esquema básico apuntado y distingamos entre simplificación administrativa y administración electrónica.

\section{SIMPLIFICACIÓN ADMINISTRATIVA}

Sobre esta materia se establecen, en primer lugar, una serie de principios generales que específicamente se califican como "orientadores de la simplificación y racionalización procedimental y organizativa" y que, desde luego, no son novedosos: "a) Orientación y servicio efectivo a la ciudadanía. b) Eficacia y eficiencia. c) Celeridad. d) Economía procedimental y organizativa. e) Racionalización y agilidad de los procedimientos administrativos y de las actividades materiales de gestión. f) Transparencia administrativa" (artículo 3.1).

Igualmente, se fijan criterios para la simplificación de procedimientos, agilización de trámites y reducción de cargas, que han de aplicarse "en el diseño y rediseño funcional de los procedimientos administrativos, así como con ocasión de su tramitación" (artículo 6.1). Se distingue entre los criterios de simplificación y agilización de los procedimientos (artículo 6.2) y los criterios de reducción de cargas y simplificación documental (artículo 6.3).

Los primeros son: "a) La unificación o eliminación de procedimientos. b) La reducción de los términos y plazos en la medida que sea posible, manteniendo todas las garantías necesarias. c) La supresión o simplificación de trámites que no aporten valor añadido o que supongan dilaciones del procedimiento, siempre que no afecten a las garantías de las personas interesadas. d) La proactividad por parte del órgano responsable del procedimiento. e) El establecimiento de modelos de declaración, memorias o test de conformidad que faciliten la elaboración de informes preceptivos. f) La extensión y potenciación de los procedimientos de respuesta inmediata o resolución automatizada para el reconocimiento inicial de un derecho o facultad, así como para su renovación o continuidad de su ejercicio; este criterio se aplicará especialmente a los procedimientos y servicios en que se resuelven las pretensiones y demandas de la ciudadanía tras un único contacto con la Administración o en un tiempo muy breve. g) La agilización de las comunicaciones. h) La desconcentración de competencias en órganos jerárquicamente dependientes. i) La orientación a la ciudadanía".

A su vez, los criterios de reducción de cargas y simplificación documental son: “a) La supresión de cargas administrativas que no sean imprescindibles para la resolución del procedimiento, eliminando requisitos no exigidos por la normativa vigente, valorando su adecuación y reduciendo la frecuencia de su aportación. b) La supresión o reducción de la documentación requerida a las personas interesadas y su 
posible sustitución por transmisiones de datos o la presentación de declaraciones responsables. c) La valoración del momento idóneo para la aportación de la documentación requerida a las personas interesadas, promoviendo siempre que sea posible, su aportación en el trámite de audiencia anterior a la formulación de la propuesta de resolución. d) El fomento de las declaraciones responsables y comunicaciones, reduciendo la aportación de datos, documentos y requisitos exigibles para el ejercicio de un derecho o el inicio de una actividad. e) La agrupación documental, incorporando en un único documento las manifestaciones que, en forma de declaraciones, certificaciones o actuaciones de similar naturaleza, haya de hacer una misma persona en un mismo trámite, o en varios si la gestión del procedimiento lo permite. f) La normalización documental, fundamentalmente de los formularios de solicitud, declaraciones responsables, comunicaciones, certificaciones y documentos de análogo carácter, diseñando modelos que faciliten y agilicen su cumplimentación, con los datos mínimos necesarios para identificar a la persona interesada y facilitando, cuando sea posible, su precumplimentación".

Por otra parte, los instrumentos de simplificación o al servicio de ésta que se regulan, de distinto alcance y naturaleza, son la memoria de cumplimiento de los principios de buena regulación en los procedimientos de elaboración de normas de la Junta de Andalucía (artículo 7), el informe en materia de simplificación y organización (artículo 8), los Planes de Calidad y Simplificación Normativa (artículo 9), el Registro de Procedimientos y Servicios (artículo 10), el Catálogo de Procedimientos y Servicios (artículo 11), los formularios (artículo 12) y el Manual para la simplificación de los procedimientos administrativos y agilización de trámites (artículo 13.1). Con carácter más general, el artículo 13.2 prevé que la Consejería competente en materia de administración pública elabore y mantenga actualizadas "otras herramientas para la simplificación de procedimientos administrativos y agilización de trámites, como guías de orientación para facilitar a los órganos directivos el análisis, diagnóstico y mejora de los procedimientos administrativos, y la elaboración de disposiciones generales que regulen nuevos procedimientos o modifiquen los ya existentes".

De este conjunto de instrumentos destacamos, en esta breve síntesis, la regulación de la memoria de cumplimiento de los principios de buena regulación en los procedimientos de elaboración de normas de la Junta de Andalucía (artículo 7), me- 
moria que el Consejo Consultivo de Andalucía venía echando en falta en los expedientes de elaboración de normas ${ }^{13}$ y que ahora resulta obligatoria ${ }^{14}$.

También deben ser destacados los Planes de Calidad y Simplificación Normativa, que entroncan con el artículo 130.1 de la Ley 39/2015 y responden al mandato incluido en el apartado segundo de la disposición adicional primera de la Ley 1/2014, de 24 de junio, de Transparencia Pública de Andalucía. Aun cuando el artículo 9 del Decreto se refiera a "Planes", en plural, se trata de un solo Plan, como prevé la Ley, que la Consejería competente en materia de administración pública debe elaborar al menos cada cuatro años, correspondiendo su aprobación al Consejo de Gobierno. El objetivo del Plan es "la revisión, simplificación y, en su caso, consolidación normativa de las disposiciones vigentes en el Derecho propio de la Comunidad Autónoma de Andalucía"; concretamente, se prevé que mediante la revisión se valore "la adopción de, al menos, las siguientes medidas: a) La reducción del número de normas. b) La adaptación de las normas a los principios de buena regulación establecidos en la legislación del procedimiento administrativo común y precisados en el artículo 7, verificando que las normas en vigor han conseguido los objetivos previstos, así como que estaban justificados y correctamente cuantificados el coste y las cargas impuestas en ellas. c) La consolidación y simplificación de la normativa vigente. d) El rediseño funcional de los procedimientos, aplicando los criterios establecidos en el artículo 6 para la simplificación de procedimientos, agilización de trámites y reducción de cargas". Adicionalmente, se contempla un informe de evaluación en el que se plasme el resultado de la ejecución de cada plan, que gozará como mínimo de la publicidad que proporciona el Portal de la Junta de Andalucía.

Igualmente, traemos a primer plano el Registro de Procedimientos y Servicios y el Catálogo de Procedimientos y Servicios. Ambos son herramientas informáticas con las que ya se contaba; el Decreto las dota, como se lee en la Memoria económica del proyecto, de 20 de octubre de 2017, "del correspondiente soporte jurídico". Mientras que el Registro no es público (artículo 10.10), el Catálogo ha de publicarse en el Portal de la Junta de Andalucía y en las sedes electrónicas (artículo 11.4), siendo "el

13 "Es cierto que el artículo 129.1 de la Ley 39/2015 dispone que en la exposición de motivos o en el preámbulo, según se trate, respectivamente, de anteproyectos de ley o de proyectos de reglamento, quedará suficientemente justificada su adecuación a dichos principios. Sin embargo, dicha declaración no es una pura formalidad, sino que debe guardar coherencia con la documentación obrante en el expediente, en la que debe quedar constancia del análisis del cumplimiento de dichos principios. En este caso, como se indica en el anterior fundamento jurídico, no existe una memoria o documento equivalente que permita considerar efectuado dicho análisis y, por ende, resulta cuestionable la declaración que se formula en la exposición de motivos" (Dictamen del Consejo Consultivo de Andalucía 286/2017, de 16 de mayo).

14 Indica la disposición adicional sexta que la memoria "se integrará en la Memoria de Análisis de Impacto Normativo a que se refiere la disposición adicional segunda de la Ley 1/2014, de 24 de junio, una vez se apruebe por el Consejo de Gobierno el decreto que la regule". 
instrumento de publicidad activa de los procedimientos administrativos a los efectos contemplados en el artículo 14.a) de la Ley 1/2014, de 24 de junio, de Transparencia Pública de Andalucía" (artículo 11.5). Ha de tenerse en cuenta que el Catálogo recoge toda la información que con carácter de mínima prevén los apartados 8 y 9 del artículo 10 para el Registro (artículo 11.2).

Tampoco supone una auténtica novedad la figura del manual para simplificación de procedimientos y agilización de trámites, dado que desde 2010 se cuenta con el denominado Manual de Simplificación Administrativa y Agilización de Trámites, aprobado por Orden de 22 de febrero del referido año.

Resta, finalmente, una breve referencia a los formularios, instrumentos que, aun a pesar de su utilidad, suscitan también recelos, pues no cabe duda de que su imposición limita la capacidad de expresión del interesado. Dejando a un lado la función que cumplen como instrumento para promover la relación electrónica con la administración (apartados 5 y 6 del artículo 12), son dos las cuestiones sobre las que se quiere llamar la atención: por una parte, la criptica redacción del primer párrafo del artículo $12.9^{15}$; y por otra, la supresión durante la tramitación del Decreto de la siguiente mención que, a nuestro juicio, hubiera fortalecido las garantías de los interesados: "Cuando se utilice en el procedimiento un formulario equivocado se requerirá a la persona interesada para que subsane, articulándose las soluciones y medios técnicos necesarios para que pueda procederse a la subsanación"16.

\section{ADMINISTRACIÓN ELECTRÓNICA}

Al igual que en materia de simplificación de procedimientos, también se establecen principios generales en materia de administración electrónica que, "en función del estado de la tecnología y con plena sujeción a las disposiciones estatales básicas en la materia", se han de tener "particularmente en consideración"; tales principios son: "a) Accesibilidad y no discriminación. b) Reducción de la brecha digital, con especial consideración a las personas mayores o con discapacidad. c) Seguridad. d)

15 "Todos los formularios y modelos específicos que tengan carácter obligatorio por establecerlo así de manera expresa la norma o acto de aprobación deberán ser publicados en el Boletín Oficial de la Junta de Andalucía. En el caso de formularios cuyo medio de presentación sea exclusivamente electrónico, se hará constar claramente que dicha publicación es únicamente a efectos informativos". A mi juicio, son dos cuestiones diferentes: una, la publicación de los formularios y modelos que sean obligatorios; y otra, el medio de presentación. No se vislumbra la razón por la que, en el caso de la presentación electrónica, la publicación del formulario sea solo a efectos informativos.

16 Artículo 12.2 del primer borrador que figura en el Portal de Transparencia, de 16 de febrero de 2018. El inciso se suprimió al ser aceptada la sugerencia en tal sentido del Gabinete Jurídico, que en su informe abogó por ello "al no guardar relación con los requisitos de los formularios, y tener un alcance de carácter más procedimental". 
Conservación. e) Interoperabilidad. f) Proporcionalidad. g) Impulso de la actuación administrativa automatizada. h) Usabilidad" (artículo 3.2). Estos principios tampoco son por completo novedosos; y ni siquiera son todos específicos del ámbito de la administración electrónica, como se puso de manifiesto en el procedimiento de elaboración del Decreto ${ }^{17}$. En realidad, la bondad de su inclusión es cuestionable dada la matización que se introduce ("en función del estado de la tecnología ... se tendrán particularmente en consideración”), pues, al cabo, en su proclamación se inserta un factor clave de debilitación del que se es muy consciente, como evidencia la expresión "con plena sujeción a las disposiciones estatales básicas en la materia".

El Decreto regula los puntos de acceso electrónico (capítulo III), la identificación y firma electrónicas (capítulo IV), el registro electrónico y las comunicaciones interiores (capítulo V), las notificaciones electrónicas (capítulo VI), los medios electrónicos en el procedimiento administrativo y la gestión documental (capítulo VII) y, agrupados en el capítulo VIII, sobre medidas organizativas, el registro electrónico de apoderamientos, los funcionarios públicos habilitados, el Registro Electrónico de Órganos Colegiados de la Administración de la Junta de Andalucía y el Registro Electrónico de Convenios de la Administración de Junta de Andalucía ${ }^{18}$.

Son muchas y variadas las cuestiones que plantea esta parte de la regulación. Destacaremos a continuación algunas de ellas.

Por lo que respecta al registro electrónico, se refuerza la opción de un único registro, el Registro Electrónico Único (artículo 26.1), en el que está llamado a integrarse el Registro Telemático Tributario (disposición transitoria cuarta) ${ }^{19}$. Cabe destacar que, de momento, solo se prevé la presentación en este registro de documentos dirigidos a las agencias públicas empresariales, agencias de régimen especial

17 Informe de la Secretaría General Técnica de la entonces Consejería de la Presidencia, Administración Local y Memoria Democrática, de 17 de mayo de 2018.

18 Se ha querido ver en este capítulo VIII una parte sustantiva de la regulación del Decreto, diferenciada de los contenidos relativos a simplificación y a administración electrónica (vid. el informe de la Dirección General de Presupuestos, de 19 de marzo de 2018, que distingue tres ejes: medidas de simplificación administrativa, régimen de la administración electrónica y medidas organizativas). A mi juicio, y como ya he indicado, el contenido del capítulo se engloba en el conjunto de preceptos dedicados a la administración electrónica.

19 El artículo 16.1 de la Ley 39/2015 prevé que los organismos públicos vinculados o dependientes de cada Administración puedan disponer de su propio registro electrónico, plenamente interoperable e interconectado con el registro electrónico general de la administración de la que dependan. Por su parte, el artículo 83 de la Ley 9/2007, de la Administración de la Junta de Andalucía, deja abierta la posibilidad de que existan distintos registros. Con anterioridad, el Decreto 183/2003, de 24 de junio, por el que se regula la información y atención al ciudadano y la tramitación de procedimientos administrativos por medios electrónicos (Internet), había optado por el Registro Telemático Único, que a la postre no era tal porque la disposición adicional 3 del propio Decreto creó al unísono el Registro Telemático Tributario. 
y los consorcios adscritos a la Administración de la Junta de Andalucía cuando "se trate de prestación de servicios o de procedimientos en los que se ejerzan potestades administrativas y así esté dispuesto en el instrumento jurídico que regule dicho procedimiento" (artículo 26.6) ${ }^{20}$.

Se establece como causa de "rechazo en el registro" de un escrito la circunstancia de que "la Administración o entidad, a la que pertenezca la unidad u órgano administrativo de destino, no se encuentre integrada en el sistema de interoperabilidad de las Administraciones Públicas" [artículo 27.4.c)]. Dado que el artículo 16.4 de la Ley 39/2015 contempla la presentación de escritos en los registros electrónicos de cualquiera de los sujetos a los que se refiere su artículo 2.1 y, en su último inciso, prescribe que "[l] os registros electrónicos de todas y cada una de las Administraciones, deberán ser plenamente interoperables, de modo que se garantice su compatibilidad informática e interconexión, así como la transmisión telemática de los asientos registrales y de los documentos que se presenten en cualquiera de los registros", no parece correcto que sea el ciudadano el que padezca las consecuencias -en forma de rechazo de su escrito- del incumplimiento de la obligación de integración en el sistema de interoperabilidad.

Por otra parte, el artículo 27.5 habilita para que "[d]e acuerdo con los instrumentos informáticos y vías de comunicación existentes, y de forma debidamente justificada, se podrán establecer limitaciones de tamaño o de cualquier otra índole a fin de garantizar la compatibilidad técnica de los documentos con las aplicaciones informáticas que se utilicen para la gestión electrónica de los servicios y procedimientos". Se trata de una previsión que claramente puede conducir a una merma de las garantías de los administrados y que, además, no tiene respaldo en ninguna ley ${ }^{21}$.

Llama también la atención el artículo 30.1.a).2º que caracteriza como notificación electrónica el acceso "por parte de funcionario público habilitado y su entrega personal cuando la persona interesada o su representante comparezca espontáneamente en las oficinas de asistencia en materia de registros y solicite la comunicación o notificación personal en ese momento". El precepto, que nos hace recordar lo es-

20 El informe de la Secretaría General Técnica de la entonces Consejería de Economía, Hacienda y Administración Pública, de 6 de noviembre de 2018, manifestó que era dudoso que pudiera excluirse la presentación de documentos dirigidos a entidades dependientes a la luz del artículo 16.1 de la Ley 39/2015. Con todo, se trata de una "solución provisional", toda vez que el apartado primero de la disposición adicional segunda prevé que "[s]in perjuicio de lo dispuesto en el artículo 26.6, la utilización del Registro Electrónico Único para los documentos propios de la actividad privada de las agencias y consorcios adscritos y de su relación con los órganos de la Administración de la Junta de Andalucía podrá realizarse una vez que se disponga de los medios electrónicos y de otra índole necesarios y se alcance el nivel adecuado de convergencia en los sistemas".

21 El Consejo Consultivo, en su dictamen de 19 de diciembre de 2019, se mostró moderadamente crítico al respecto. 
tablecido en el artículo 22 de la Ley 11/2007, de 22 de junio, de acceso electrónico de los ciudadanos a los servicios públicos, se aparta del artículo 41.1.a) de la Ley 39/2015, que caracteriza como una notificación practicada por medios no electrónicos la realizada "con ocasión de la comparecencia espontánea del interesado o su representante en las oficinas de asistencia en materia de registro", habiendo solicitado "la comunicación o notificación personal en ese momento". Por otra parte, la práctica de la notificación mediante su "[p]uesta a disposición en la sede electrónica o dirección electrónica habilitada de otras Administraciones Públicas, previa firma del correspondiente convenio por parte de la Consejería competente en materia de transformación digital" es un medio que no se encuentra contemplado en la Ley $39 / 2015$.

También debemos destacar el artículo 33, que regula la comunicación previa y notificación a personas obligadas a relacionarse por medios electrónicos en procedimientos iniciados de oficio. El precepto parte del alta de oficio en el sistema de notificaciones electrónicas de la Administración de la Junta de Andalucía cuando se conozcan los datos para realizar el aviso de notificación (apartado primero). Y concilia satisfactoriamente la obligatoriedad de la relación por medios electrónicos con las garantías del administrado, en los procedimientos iniciados de oficio, en los casos en que no se conocen su dirección de correo electrónico o los datos del dispositivo electrónico a los efectos de recibir los avisos de notificaciones electrónicas. Al efecto, distingue entre la apertura de un periodo de información o actuaciones previas al inicio de oficio de un procedimiento (apartado segundo) y la primera notificación del procedimiento, en el supuesto de que no hayan mediado la apertura de un periodo de información o actuaciones previas (apartado tercero). Para el primer caso, se dispone que se podrá enviar al interesado sobre el que pese la obligación de relacionarse electrónicamente "una comunicación en papel con un aviso previo recordándole su deber de relacionarse por medios electrónicos y la necesidad de darse de alta en el sistema de notificaciones electrónicas de la Administración de la Junta de Andalucía, indicándole cómo proceder a su alta y manifestándole que dispone para ello de un plazo de diez días, incorporando sus datos para la remisión de avisos"; añade el apartado segundo que "[s]e le advertirá que, transcurrido dicho plazo sin que haya procedido a ello, se le dará de alta de oficio en el sistema de notificaciones electrónicas de la Administración de la Junta de Andalucía sin datos para la remisión de avisos, y que en lo sucesivo podrá ser utilizada para la práctica de notificaciones electrónicas en todos los procedimientos en que tenga condición de persona interesada" ${ }^{22}$. Para

22 Obviamente, en los supuestos en los que sea obligada la relación electrónica, pues es posible que ello suceda solo respecto de ciertos procedimientos. Vid. esta observación en el informe de la Secretaría General Técnica de la entonces Consejería de la Presidencia, Administración Local y Memoria Democrática, de 17 de mayo de 2018. 
el segundo caso, se establece que "el órgano gestor del procedimiento realizará la primera notificación del procedimiento en papel, incluyendo los contenidos de dicho aviso" (el aviso de notificación electrónica). Lógicamente, se exceptúa de estas prescripciones el supuesto en el que "el inicio del procedimiento se produzca mediante convocatoria publicada en el Boletín Oficial de la Junta de Andalucía, a la que deban concurrir las personas interesadas presentando obligatoriamente escritos de solicitud por medios electrónicos"; la razón de ello es que "el alta en el sistema de notificaciones electrónicas de la Junta de Andalucía se realizará de oficio con los datos que figuren en la solicitud" (apartado cuarto).

También merece una valoración positiva el apartado 3.c) del artículo 35, que advierte expresamente que "[p]odrá accederse al contenido de las notificaciones rechazadas con efectos meramente informativos", de modo que se impide la criticable práctica consistente en impedir el acceso a la notificación rechazada. No sucede lo mismo respecto de la regulación de las denominadas notificaciones caducadas, que son aquellas que tienen "por destinataria una persona no obligada a relacionarse por medios electrónicos que no haya elegido voluntariamente este medio", cuando ha "transcurrido el plazo previsto en la normativa básica para practicar la notificación sin que la persona interesada haya accedido a su contenido"; estas notificaciones caducadas "no estarán disponibles para su acceso, sin que por ello se entiendan por rechazadas" [apartado 3.d)]; en principio, no hay razón que justifique la privación del acceso a estas notificaciones, máxime teniendo en cuenta que se prevé que permanezcan en el sistema un mínimo de cinco años (artículo 35.5) ${ }^{23}$. Por otra parte, resulta acertada, en líneas generales, la previsión de que "[a]ntes de que la persona destinataria acceda al contenido de la notificación, se le indicará que puede rechazarla, con la advertencia de que, en tal caso, se entenderá practicada" (apartado cuarto); ello no obstante, se podría haber previsto también la obligación de advertir que no hay obligación -valga la redundancia- de acceder a la notificación cuando no se está obligado a relacionarse electrónicamente. Finalmente, se podría haber mejorado el contenido del apartado quinto si se hubiera explicitado que la eliminación de las notificaciones del sistema no implica, como es natural, la eliminación del archivo electrónico ${ }^{24}$.

23 A juicio de la entonces Dirección General de Planificación y Evaluación, no es necesario que las notificaciones permanezcan accesibles en el sistema porque los interesados ya disponen de ellas en formato papel (vid. la valoración del informe emitido por la Secretaría General Técnica de la Consejería de Economía, Hacienda y Administración Pública, de 19 de diciembre de 2018). Sin embargo, aunque no sea necesario, sí puede resultar conveniente.

24 Así se puso de manifiesto en el informe de la Secretaría General Técnica de la entonces Consejería de Economía, Hacienda y Administración Pública, de 6 de noviembre de 2018, con referencia al expediente electrónico. 
Por lo que respecta a la carpeta ciudadana (artículo 38), se trata de una herramienta que ya existía, de modo que valoramos positivamente que ahora se regule, imponiéndose determinados contenidos mínimos (apartado 2$)^{25}$.

Finalmente, otro precepto sobre el que procede llamar la atención es el artículo 39, que recuerda que "[e]l deber de relacionarse por medios electrónicos no podrá imponerse a través de actos administrativos generales de convocatoria”. En realidad, el deber no puede imponerse a través de actos administrativos, ya sean generales o singulares.

\section{EPÍLOGO}

Como ya se advirtió al comienzo de estas páginas, el Decreto 622/2019, de 27 de diciembre, de administración electrónica, simplificación de procedimientos y racionalización organizativa de la Junta de Andalucía, ocupa un lugar destacado en el ámbito del Derecho Administrativo propio de la Comunidad Autónoma. Coincidimos con el Consejo Consultivo en que, prácticamente en su totalidad, se relaciona con el derecho a la buena administración, con una proyección directa sobre el cumplimiento de los deberes y el ejercicio de los derechos de los ciudadanos.

Con más luces que sombras, supone una puesta al día de la regulación de la materia y un avance en el desarrollo de los principios que proclama el artículo 133.1 del Estatuto de Autonomía y el artículo 3 de la Ley 9/2007, destacando en particular el desarrollo y concreción que ofrece de los aspectos básicos y de procedimiento común regulados en las Leyes 39 y 40 de 2015. Sea, pues, bienvenido el nuevo Decreto, del que hemos ofrecido una primera aproximación, conscientes de que merece una mayor atención por el alcance de la regulación que contiene.

25 Son los siguientes: "a) Consulta al estado de tramitación y, en su caso, acceso al contenido de los expedientes de los procedimientos administrativos y servicios. b) Consulta de solicitudes o escritos presentados en el Registro Electrónico Único de la Administración de la Junta de Andalucía. c) Acceso a las notificaciones electrónicas. d) Consulta de la información personal existente en las Administraciones Públicas previa autorización de la persona interesada. e) Acceso a certificados, tarjetas y carnés expedidos por la Administración de la Junta de Andalucía, sus agencias y, en su caso, consorcios adscritos, que tendrán la consideración de copia electrónica auténtica". Se trata de contenidos mínimos porque el precepto incluye la siguiente cláusula abierta: "Cualquier otra que se estime de utilidad para mejorar los servicios que la Administración de la Junta de Andalucía, sus agencias y, en su caso, consorcios adscritosprestan a la ciudadanía”. Es de desear que en el futuro se facilite que los titulares de las carpetas puedan poner a disposición de la Administración, sin necesidad de que se esté tramitando un procedimiento administrativo concreto, documentación que consideren relevante, al efecto de que dicha documentación pueda considerarse obrante en el poder de la Administración en los términos del artículo 28.2 de la Ley 39/2015. 